Pharmaceutical companies may have a genuine interest in education, but should that allow them to influence prescribing? Research has shown that although they believe their own prescribing is unaffected, many doctors believe that their colleagues are influenced (Halperin et al, 2004). Vassilas \& Matthews call for trusts to develop guidelines and this is essential. I believe we also need to take individual responsibility. In particular, we need to look at our education and how we fund it. We can all buy lunch and pens for ourselves, but realistically how many of us could attend big international symposia if not sponsored? Clearly that should not be the case. I have read suggestions that pharmaceutical companies with a real interest in education could contribute anonymously to a general fund for education without any payback. This is something to consider. For myself I have found I can access excellent local and online education within the scope of a National Health Service consultant budget and will continue not to see representatives of pharmaceutical companies or accept their gifts.

HALPERIN, E. C., HUTCHISON, P. \& BARRIER, R. C. jR (2004) A population-based study of the prevalence and influence of gifts to radiation oncologists from pharmaceutical companies and medical equipment manufacturers. International Journal of Radiation Oncology and Physics, 59, 1477-1483.

Winnie Manning Consultant in Old Age Psychiatry, Roadmeetings Hospital, Carluke, Lanarkshire, Scotland

email:Winnie.manning@lanarkshire.scot.nhs.uk

\section{Functionalised community mental health teams and in-patient care}

Commander \& Disanyake (Psychiatric Bulletin, 30, 213-215) reported that the number of psychiatric in-patients in west Birmingham fell by one-third between 1992 and 2003 and attributed this change to functionalised community mental health teams. They have not discussed several other factors which I think are also relevant.

In 1992 there were about 5 consultant (adult) psychiatrists supported by about 10 junior doctors and a small number of nurses and social workers in the community, serving an inner-city population of about 250000 . By 2003 the number of mental health professionals, including doctors, had greatly increased (probably tripled) and west Birmingham had become part of the Birmingham and Solihull Mental Health Trust, covering a population of about 1.2 million. The large psychiatric hospital in west Birmingham had closed and was replaced by fewer admission wards and fewer continuing care wards. Several 'respite hostels' were set up by the trust for patients who would have been admitted to hospital in the past, and residents may not have been counted as admissions. Beds were often full, and patients were sometimes moved to othe psychiatric hospitals and occasionally to the private hospital in Birmingham.

With fewer beds, patients with depression and other psychiatric illnesses with less challenging behaviour who did not require admission under the Mental Health Act 1983 received out-patient care. This may explain the increase in the proportion of compulsorily detained patients. Wards were often full, with occupancy rates in excess of $100 \%$ most of the time.

I wonder if the fall in the number of people in hospital was not only due to the functionalised teams but also because there were fewer beds, more staff working in the community and other places for patient admission.

Seng E. Goh Consultant in Old Age Psychiatry, Bushey Fields Hospital, Bushey Fields Road, Dudley DY1 2LZ

\section{Risk assessment in self-harm}

Lepping et al (Psychiatric Bulletin, May 2006, 30, 169-172) report an increase in the number of psychosocial assessments following the introduction of a self-harm pathway. Questions remain about patients who abscond from the accident and emergency department (A\&E). Factors that may lead to qualitative improvement in assessments should be given further consideration.

An integrated care pathway between the local university hospital and the mental health trust was set up in Middlesbrough in February 2004. Doctors in A\&E use a modified SAD PERSONS scale for triage (Hockberger \& Rothstein, 1998). Patients scoring $<5$ are offered home visits by the self-harm team and those scoring $\geqslant 5$ are admitted to the medical assessment unit for daytime assessments the following day. Six-month data for comparable months in 2003 and 2004 have shown a $24 \%$ increase in referrals, a $17.8 \%(P<0.0001)$ increase in assessments and a reduction in the number of absconsions/self-discharges by $81.5 \%$ $(P<0.0001)$. Admissions to the medical assessment unit have increased by $33 \%$; $42 \%$ of planned home visits were cancelled by patients. These were from a group who had a low-risk score in triage.

The modified SAD PERSONS scale is easy to use by non-psychiatrists and allows reliable risk assessment of selfharm to be carried out at an early stage, which guides professionals if patients refuse treatment or abscond. We consider that daytime assessments after patients have had time to recover from the effects of drugs, alcohol or overdose, which allow access to collateral information, are of further benefit.

HOCKBERGER, R. S. \& ROTHSTEIN, R. J. J. (1988) Assessment of suicide potential by nonpsychiatrists using the SAD PERSONS score. Journal of Emergency Medicine, 6, 99-107.

*Madhuri Rajwal Locum Consultant in Crisis Resolution, Southwest Yorkshire Mental Health Trust, Manygates, Belle Isle Health Park, Portobello Road, Wakefield WF1 5NJ,

email: madhuri.rajwal@swyt.nsh.uk,

Amanda Gash Consultant in Liaison Psychiatry Tees, Esk and Wear Valleys NHS Trust 